\title{
In-Situ Analysis of Heat Accumulation during Ultrashort Pulsed Laser Ablation
}

\author{
Benedikt Bornschlegel ${ }^{1}$, Jana Köller ${ }^{1}$ and Johannes Finger ${ }^{2}$ \\ ${ }^{1}$ Chair for Laser Technology LLT, RWTH Aachen University, Germany \\ E-mail: benedikt.bornschlegel@llt.rwth-aachen.de \\ ${ }^{2}$ Fraunhofer Institute for Laser Technology ILT, Germany
}

\begin{abstract}
Ultrashort pulse (USP) laser processing with pulse durations below 10 ps enables laser structuring with good surface quality, highest precision and low thermal load for the processed workpieces. However, when it comes to scale up the productivity of such processes by increasing the average power, accumulation of the remaining residual energy gets relevant and can influence the processing results significantly. For instance, heat accumulation can induce distinct melt layers. Thus the melt-free, high precision ablation, which is the main advantage of USP processing, is compromised. This is especially pronounced for high repetition rate processes. For a better insight into this effect, the development of the workpiece temperature during USP ablation processes is measured with a thermographic system. With this setup, the residual energy is determined and changes of the heat input during the processing time are identified. These changes are attributed to changes of the surface roughness during the processes within this work. Furthermore, the validity of simple physical model considerations is discussed.
\end{abstract}

DOI: $10.2961 /$ jlmn.2020.01.2010

Keywords: ultrashort pulsed ablation, heat accumulation, residual heat, high repetition rate, thermographic, in-situ, ultrafast laser ablation

\section{Introduction}

Material ablation with ultrashort pulsed (USP) laser radiation with pulse durations below 10 ps enables structuring with better surface quality, higher precision and less thermal impact on the workpiece compared to longer pulsed laser radiation [1]. However, due to the low productivity USP processing is currently only established for very few industrial applications [2-4]. In order to improve the productivity and shorten the process times, the utilization of high power ultrafast laser systems with average powers in the range of hundreds of Watt is a current topic of research in the field of laser ablation. Due to the limitation of the applicable single pulse energy through the often reported optimum fluence for USP metal ablation [5,6], the high average power must be put into effect by other approaches [7]. The three most common approaches for this purpose are the use of high repetition rates $[2,8]$, pulse bursts $[4,9,10]$ or multi-beam optics [7]. Within the multi-beam approach the high pulse energy provided by high power laser sources is split into multiple partial beams. Thus, each individual spot reaches the optimum fluence. The approach of using high repetition rates is in need for fast beam deflection to ensure a sufficiently small spatial pulse overlap. In contrast, for the pulse burst approach conventional galvanometer scanners can be applied. The approach of pulse burst consist of replacing the single pulses by short pulse trains. For high repetition rates and pulse bursts the temporal and spatial distance of the consecutive pulses are so small that interaction between subsequent pulses cannot be neglected. These interactions are namely shielding effects of plasma and particles and heat accumulation of the residual heat. This affects the quality [11] and achieved ablation efficiency [3] of these processes significantly. The heat accumulation of residual energy of the applied laser pulses can locally reach the melting temperature of the material at the surface of the workpiece. Melting of the surface has beneficial aspects in cases where a smooth surface finish is desired, but always impairs the process precision and therefore the main advantage of ultrafast laser ablation. Several studies investigated this effect to gain an understanding of the percentage share of the irradiated energy which remains as heat in the workpiece and contributes to the thermal load $[3,11,12]$. The work presented here is intended to verify and support already reported results by investigating the heating effect in a time-resolved manner. Therefore, the temperature development of the workpiece during USP ablation processes is measured with a thermographic system. With this setup a percentage estimation of the residual heat by comparison with a simulation as well as the identification of changes of the heat input during the process time are possible. Furthermore, the experimental results are compared to a simple physical model.

\section{Theoretical considerations}

For a theoretical estimation of the residual heat that is deposited into the workpiece an ablation model depending on a threshold fluence $F_{\text {th }}$ introduced by Nolte et al. [1] can be used. Within this model the ablation depth $\Delta \mathrm{z}$ is given by

$$
\Delta z=\delta \ln \left(\frac{F}{F_{t h}^{\delta}}\right) \quad(\delta \gg l)
$$




$$
\Delta z=l \ln \left(\frac{F}{F_{t h}^{l}}\right) \quad(\delta \ll l)
$$

Where $\delta$ is the optical penetration depth and $l$ the electron heat penetration depth of the material and $F_{t h}^{\delta}$ or rather $F_{t h}^{l}$ is the corresponding threshold fluence and $F$ is the absorbed fluence. Equation (1) describes the ablation depth for a low fluence regime where the optical penetration depth defines the energy absorption. Equation (2) can be applied for a high fluence regime where the electron heat conductivity dominates the energy distribution. For further consideration the ablation depth is written as

$$
\begin{aligned}
& \Delta z=\delta_{E} \ln \left(\frac{F}{F_{t h}}\right) \\
& F(r)=F_{0} e^{\left(\frac{-2 r^{2}}{w_{0}^{2}}\right)}
\end{aligned}
$$

With a general energy penetration depth $\delta_{E}$ and a threshold fluence $F_{t h}$. Equation (4) describes a Gaussian shaped beam profile where $F_{0}$ is the peak fluence in the center of the beam and $w_{0}$ is the beam radius. Thus, the ablation volume of each pulse can be calculated as a volume-integral of equation (3) with equation (4) as shown by Račiukaitis et al. [5] to

$$
V=\frac{\delta_{E} \pi w_{0}^{2}}{4} \ln ^{2}\left(\frac{F_{0}}{F_{t h}}\right)
$$

Taking the pulse energy $E_{P}=\frac{F_{0} \pi w_{0}^{2}}{2}$ and the repetition rate $f_{\text {rep }}$ of the laser pulses into account, the ablation efficiency can be described as $[5,8]$

$$
\frac{\dot{V}}{\bar{P}}=\frac{1}{2} \frac{\delta_{E}}{F_{0}} \ln ^{2}\left(\frac{F_{0}}{F_{t h}}\right)
$$

Here, $\bar{P}$ is the average output power applied. Equation (6) directly leads to the efficiency maximum for $F_{0, \max }=$ $e^{2} F_{t h}$ which can be confirmed experimentally [5,8]. The boundary radius $r_{B}$ of the ablation volume can be calculated by equating equation (4) with $F_{t h}$ to

$$
r_{B}=\sqrt{\frac{w_{0}^{2}}{2} \ln \left(\frac{F_{0}}{F_{t h}}\right)}
$$

The residual energy that does not participate in the ablation of each applied laser pulse is described with formula (8) as a direct consequence of the threshold model [7].

$$
E_{r e s}=r_{B}^{2} \pi F_{t h}+\iint_{0}^{2 \pi} \int_{r_{B}}^{\infty} F_{0} e^{\left(\frac{-2 r^{2}}{w_{0}^{2}}\right)} d \varphi d r
$$

The amount of residual energy is visualized in Fig. 1.

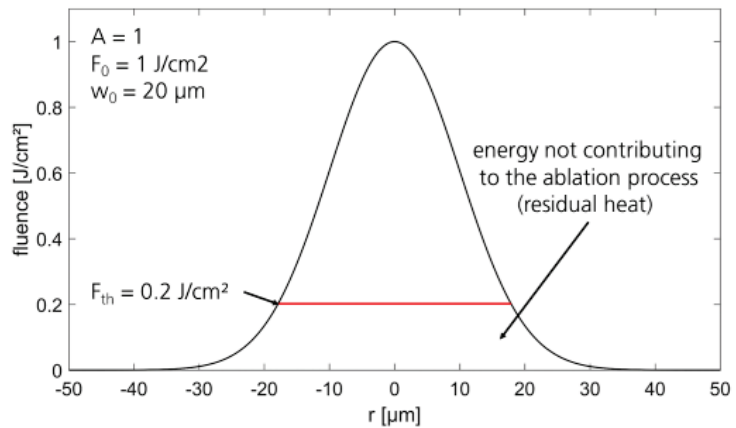

Fig. 1 Schematic representation of the spatial fluence distribution and the residual heat resulting from the threshold fluence (Absorption $\mathrm{A}$, applied fluence $\mathrm{F}_{0}$, beam radius $\mathrm{w}_{0}$, threshold fluence $\mathrm{F}_{\text {th }}$ ).
The factor $\eta_{\text {res }}=\frac{E_{\text {res }}}{E_{P}}$ is defined as the ratio between residual energy and incident pulse energy [7]. For the simulation in this work a heat source with $P_{\text {heat }}=\eta_{\text {res }} \cdot \bar{P}$ is used, where $\bar{P}$ is the average output laser power. Considering this, a constant energy input during the process can be expected, if the fluence is not varied. In addition, the total amount of residual heat per pulse depends on the ratio between the threshold fluence and the applied single pulse peak fluence [7]. The closer the fluence is to the threshold, the larger is the energy share that will remain in the workpiece without participating to the ablation process.

\section{Experimental setup and procedure}

The experiments in this work are carried out with an Amphos 400 high power laser system. The emitted laser radiation is focused with an f-theta objective with a focal length of $160 \mathrm{~mm}$. For the beam deflection an intelliSCAN 14 from Scanlab $\mathrm{GmbH}$ is utilized. The spot diameter on the sample surface is $2 w_{0}=60 \mu \mathrm{m}\left(1 / \mathrm{e}^{2}\right)$. The laser system emits pulses with a central wavelength of $\lambda=1030 \mathrm{~nm}$ with variable pulse durations between $\tau=2-20 \mathrm{ps}$. In this work all experiments are carried out with a pulse duration of $\tau=2 \mathrm{ps}$. The repetition rate is adjustable between $f_{\text {rep }}=0.4-40 \mathrm{MHz}$ with an average power of $\bar{P}=400 \mathrm{~W}$. Lower repetition rates are obtained by an integrated pulse picker unit. The within this processes achieved surface morphology is evaluated with a laser scanning microscope (LSM) Keyence VK-9700 from Keyence Corporation.

For the in-situ investigation of the temperature change during these processes an ImageIR ${ }^{\circledR} 8300$ from InfraTec $\mathrm{GmbH}$ is synchronized with the laser source by a pulse generator of the 9200 Series from Quantum Composers Inc. as shown in Fig. 2. A germanium filter in front of the camera objective protects the thermographic system from the laser radiation. Germanium is used due to its transparency in the wavelength range of the camera and its absorption at the laser wavelength.

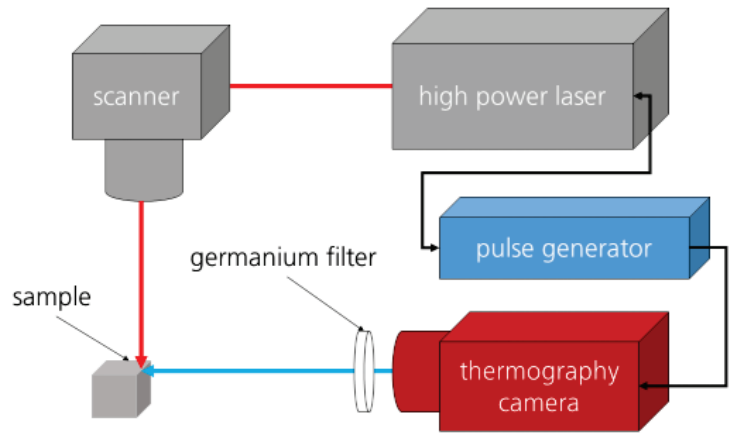

Fig. 2 Setup for in-situ investigation of the heat accumulation of ablation processes.

The whole process is monitored in the side view of the sample as depicted in Fig. 3 to gain a depth profile of the temperature. To minimize undesired reflections and scattered radiation of the surrounding, the side area of the sample is sandblasted and the process chamber is darkened. The thermographic system is calibrated to the emissivity of the sample surface in a temperature range of $40^{\circ} \mathrm{C}$ to $180^{\circ} \mathrm{C}$. As the calibration is only possible down to a temperature difference of the sample to the surrounding of round about 
$20 \mathrm{~K}$, measured temperatures below $40{ }^{\circ} \mathrm{C}$ must be interpreted with caution. Also, the decay behavior of the temperature cannot be evaluated. For temperature evaluation over time a point $0.82 \mathrm{~mm}$ below the sample edge (Fig. 3 ) is evaluated in order to exclude influences of the hot ablation products above the surface. For the temperature evaluation from the recorded images the pixel in the mentioned depth and the adjoining pixels are averaged and plotted over the process time. The scanning strategy is kept equal for all processes. As depicted in Fig. 3, the lines are scanned bidirectional parallel to the sample edge in a $3 \times 3 \mathrm{~mm}^{2}$ field with a scan speed of $v_{s}=3.5 \mathrm{~m} / \mathrm{s}$ and a line pitch of $\Delta y=18 \mu \mathrm{m}$ and the respective number of layers (Table 1 ).

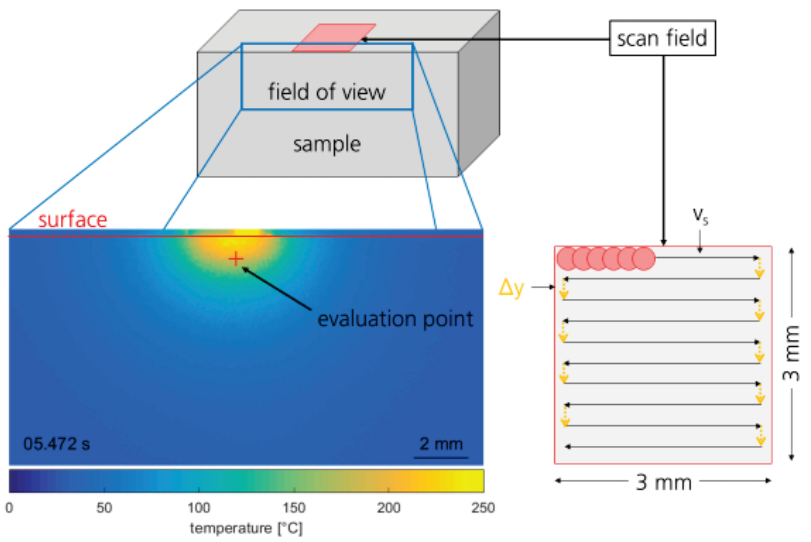

Fig. 3 Schematic sample setup with the point of view, the evaluation point for the investigation over time and the scan strategy with illustrated scan direction and y pitch on the sample surface.

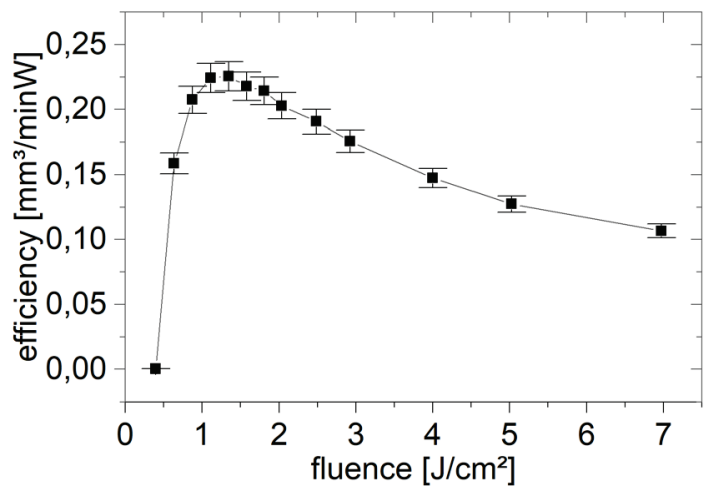

Fig. 4 Efficiency study for stainless steel 1.4301 with a maximum for $\quad F_{0} \approx 1 \mathrm{~J} / \mathrm{cm}^{2} . \quad\left(f_{\text {rep }}=400 \mathrm{kHz}, \quad 2 w_{0}=36 \mu \mathrm{m}, \quad \tau=2 \mathrm{ps}\right.$, $\lambda=1030 \mathrm{~nm})$

To keep the measured temperature rise directly comparable between different processes, the processes are carried out with the same average power, scan speed and line pitch. Only the repetition rate and therefore the single pulse peak fluence is varied. Two measurement series with a low and a high average power are carried out for stainless steel. To obtain the optimum fluence for single pulse ablation $[5,8]$ for a low as well as for a high repetition rate, the average power is adjusted. To determine good processing conditions, the ablation efficiency for the stainless steel 1.4301 is determined by a pretest. Within this pretest the ablated volume of a $3 \times 3 \mathrm{~mm}^{2}$ processing field is measured and related to the applied average power. The obtained efficiency is plotted versus the fluence in Fig. 4. Due to these results the power is set to $8.5 \mathrm{~W}$ and $70 \mathrm{~W}$ which corresponds to an optimum fluence at $800 \mathrm{kHz}$ and $6500 \mathrm{kHz}$, respectively. The number of passes is chosen that the same energy per area is applied to the material, resulting in 187 passes at $8.5 \mathrm{~W}$ and 23 passes at $70 \mathrm{~W}$. All process parameters are listed in Table 1.

Table 1 Summary of the used parameter

\begin{tabular}{ll}
\hline \multicolumn{2}{c}{ process parameter } \\
\hline wavelength $\lambda$ & $1030 \mathrm{~nm}$ \\
focus diameter $2 w_{0}$ & $60 \mu \mathrm{m}$ \\
pulse duration $\tau$ & $2 \mathrm{ps}$ \\
scan speed $v_{s}$ & $3.5 \mathrm{~m} / \mathrm{s}$ \\
line pitch $\Delta y$ & $18 \mu \mathrm{m}$ \\
repetition rate $f_{\text {rep }}$ & $0.2-6.5 \mathrm{MHz}$ \\
average power $\bar{P}$ & $8.5 \mathrm{~W} ; 70 \mathrm{~W}$ \\
single pulse fluence $F_{0}=\frac{2 \bar{P}}{f_{\text {rep }} \pi w_{0}^{2}}$ & $0.1-25.0 \mathrm{~J} / \mathrm{cm}^{2}$ \\
number of passes & $187 ; 23$ \\
\hline
\end{tabular}

\section{Experimental results}

The temperature development over time for processes with $8.5 \mathrm{~W}$ and $70 \mathrm{~W}$ average power and the six varied repetition rates are shown in Fig. 5 a)-c) and Fig. 6 a)-c). The individual temperature maxima, when the scanning process reaches the sample edge, cannot be resolved in Fig. 5 due to the high number of passes for $8.5 \mathrm{~W}$ (187), which leads to an optical widening of the measuring line. For the $70 \mathrm{~W}$ processes in Fig. 6 the individual temperature maxima caused by the scanning process (heat accumulation between laser passes), are clearly distinguishable and represent the 23 scanned layers.

For the lowest repetition rates $200 \mathrm{kHz}$ and $400 \mathrm{kHz}$ in Fig. 5 a) an nonuniform temperature rise at the beginning of the processes is observable. Later, the temperature adjusts to a saturated course, where the losses due to heat diffusion into the bulk and the energy input are in equilibrium. Nevertheless, due to the temperature calibration the absolute values around $30{ }^{\circ} \mathrm{C}$ may vary. However, the relative curve progression with the uneven rise at the beginning may still be a qualitative estimation for a change of the thermal input (i.e. non constant factor $\eta$ ) during the process. The corresponding processes for $70 \mathrm{~W}$ average power with the lowest repetition rates in Fig. 6 a) behave similar to the two corresponding low power processes. The thermal input increases during the process time and the saturation of the temperature rise at the end of the process begins.

In Fig. $5 \mathrm{~b}$ ) the temperature for the low power process with $800 \mathrm{kHz}$ with a resulting fluence next to the optimum and the process with $1600 \mathrm{kHz}$ is plotted. Both curves show no evidence of the change in the thermal input as mentioned before but the process with the lower fluence shows an at about $10^{\circ} \mathrm{C}$ higher saturation temperature compared to the $800 \mathrm{kHz}$ process. The resulting fluences of the corresponding high power processes in Fig. $6 \mathrm{~b}$ ) are still above the optimum. Both processes show an increase in the thermal input at the beginning. A similar end temperature to the high power processes with lower repetition rates is obtained. A flattening of the curve for $1600 \mathrm{kHz}$ can be suspected. 

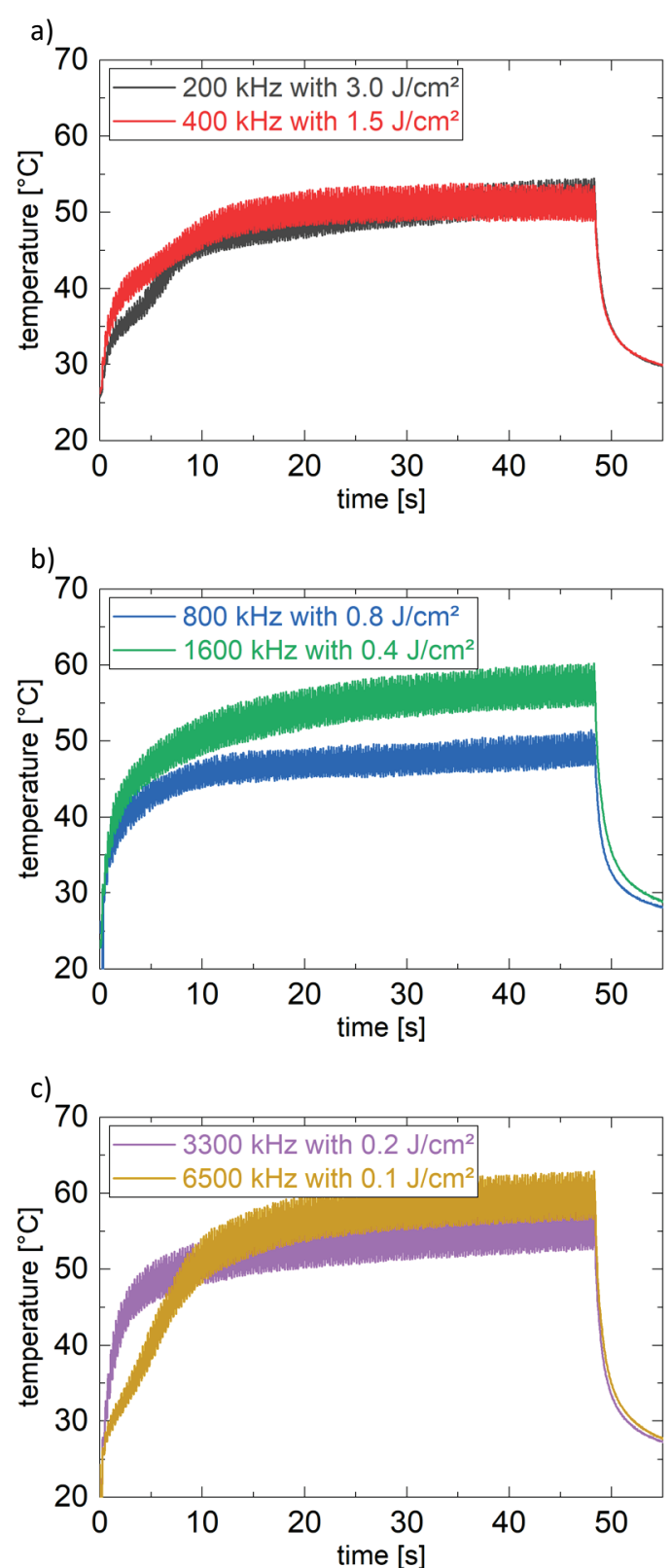

Fig. 5 Temperature development $0.82 \mathrm{~mm}$ below the surface for processes with $8.5 \mathrm{~W}$ average power for stainless steel with $200 \mathrm{kHz}$ (a), $400 \mathrm{kHz}$ (a), $800 \mathrm{kHz}$ (b), $1600 \mathrm{kHz}$ (b), $3300 \mathrm{kHz}$ (c) and $6500 \mathrm{kHz}$ (c) repetition rate with corresponding single pulse fluence.

For the low average power with $3300 \mathrm{kHz}$ in Fig. $5 \mathrm{c}$ ) also no thermal input change but a higher saturation temperature comparable with the $1600 \mathrm{kHz}$ process in Fig. $5 \mathrm{~b}$ ) is obtained. The process with the highest repetition rate at low power on the other hand shows a similar thermal behavior to the two processes in Fig. 5 a). The high power processes with high repetition rates reaching the measured optimum fluence (Fig. $6 \mathrm{c}$ )) show no change in the thermal behavior (i.e. a smooth temperature rise). A saturation temperature is almost reached. Also the end temperature is $45^{\circ} \mathrm{C}$ to $70{ }^{\circ} \mathrm{C}$ below the end temperatures of the four processes with lower repetition rate and higher fluence.

Compared to each other it becomes evident that the reached temperatures of the high power processes are 40 to $100{ }^{\circ} \mathrm{C}$ above the corresponding low power processes. But
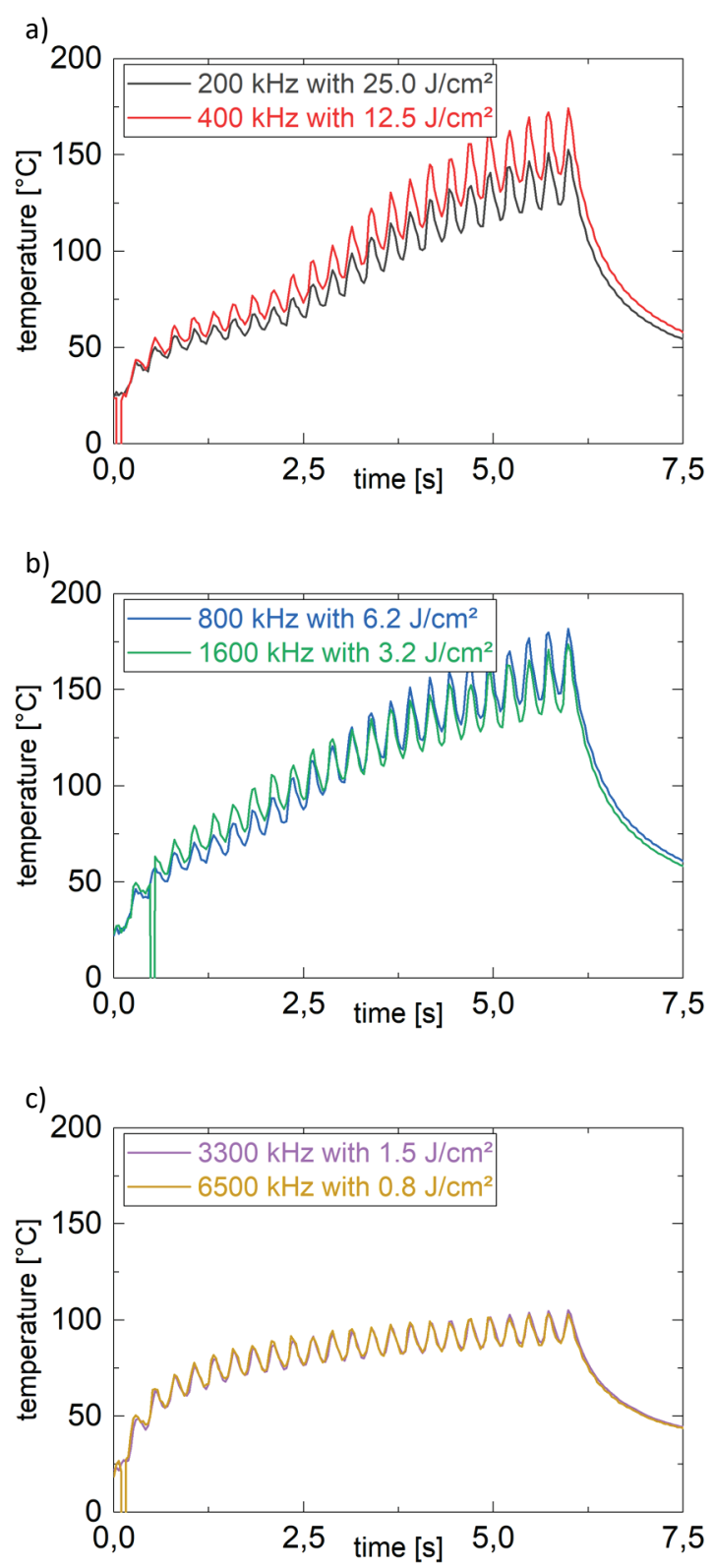

Fig. 6 Temperature development $0.82 \mathrm{~mm}$ below the surface for processes with $70 \mathrm{~W}$ average power for stainless steel with $200 \mathrm{kHz}$ (a), $400 \mathrm{kHz}$ (a), $800 \mathrm{kHz}$ (b), $1600 \mathrm{kHz}$ (b), $3300 \mathrm{kHz}$ (c) and $6500 \mathrm{kHz}(\mathrm{c})$ repetition rate with corresponding single pulse fluence.

for both power regimes a change in the thermal behavior is observed if the fluence deviates strongly from the measured optimum fluence. Furthermore, the increase in residual heat predicted by the model due to decreasing fluence may be observed in the end temperature of the low power processes with applied fluences below $0.8 \mathrm{~J} / \mathrm{cm}^{2}$. But for fluences higher than $0.8 \mathrm{~J} / \mathrm{cm}^{2}$, where the change in the thermal behavior occurs, the end temperature is contrary to the predictions also rising. The interpretation for high power processes is similar where the temperature for the four processes with the highest fluence starts below the processes with the lowest fluence, but overtakes them after the change in the thermal input. 
For further investigation a finite element simulation with COMSOL Multiphysics is carried out. Due to the calculation time only for the high power processes with shorter process time the simulation can be done with reasonable expense. For the simulation some simplifications have to be assumed. First, a continuous surface heat source with the Gaussian shape of the focus and $30 \%$ of the average power is scanned across the sample surface as displayed in Fig. 3. Second, the material properties are kept constant to literature values and the boundaries are assumed to be perfectly isolated. For a good comparability the temperature in the same evaluation spot as marked in Fig. 3 is used for the temperature evaluation that is plotted in Fig. 7.

As expected, the simulated temperature increases in the beginning and slows down approaching to a saturation temperature afterwards. It is evident that the simulation and the experimental process with $6500 \mathrm{kHz}$ show the same course and almost the same average temperature. Therefore, a $\eta$ res $\approx 0.3$ can be assumed for this process next to the optimum fluence. However, in a process with $6500 \mathrm{kHz}$ repetition rate and high spatial pulse overlap shielding effects of the ablation plumes to the subsequent pulses are unavoidable. This can lead to a reduced absorbed fluence and also to secondary heating effects by the ablation products. In contrast, the temperature of the process with $200 \mathrm{kHz}$ is below the simulated values at the beginning of the process. But after 3 seconds of the process when the change of the thermal input occurs the temperature rises faster and overtakes the simulation. Contrary to the common opinion that high repetition rates lead to higher temperatures this result shows that residual heat and not the repetition rate is the key factor that determines the thermal load. This is visualized in Fig. 8, where the highest measured temperature during the processes is shown as a function of the applied repetition rate for the process with $70 \mathrm{~W}$ average power. The energy input for all repetition rates is the same and the highest temperature is reached for the process applying $800 \mathrm{kHz}$.

For further investigation of these thermal behavior, exsitu experiments are carried out. Therefore, the processes with two different repetition rates for $8.5 \mathrm{~W}$ and $70 \mathrm{~W}$ are repeated 18 times and 22 times, respectively. To investigate the surface development during the process the processes are stopped after a different number of passes. The surface roughness is measured and microscopic images are taken after each scanned layer of the high power process and after every tenth pass of the low power process. The temporal evolution of the roughness, the temperature and the surface of the process with $70 \mathrm{~W}$ average power and $200 \mathrm{kHz}$ repetition rate is depicted in Fig. 9. An observable change in the thermal behavior is shown. In Fig. 10 the roughness, temperature and surface of a process with $70 \mathrm{~W}$ and $6500 \mathrm{kHz}$ is depicted without this change.

At point 1 in Fig. 9 at the beginning of the process a smooth surface roughness $S a$ and $S z$ is presented. Directly before the measured thermal change sporadic melt structures begin to form on the surface and are also recognized in the surface roughness (point 2). When the temperature increase weakens slightly (from point 3 onwards), the increase in roughness slows down and the uneven melt structures cover the whole surface. At the end of the process (point 4) even melt structures cover the whole sample surface. Therefore,

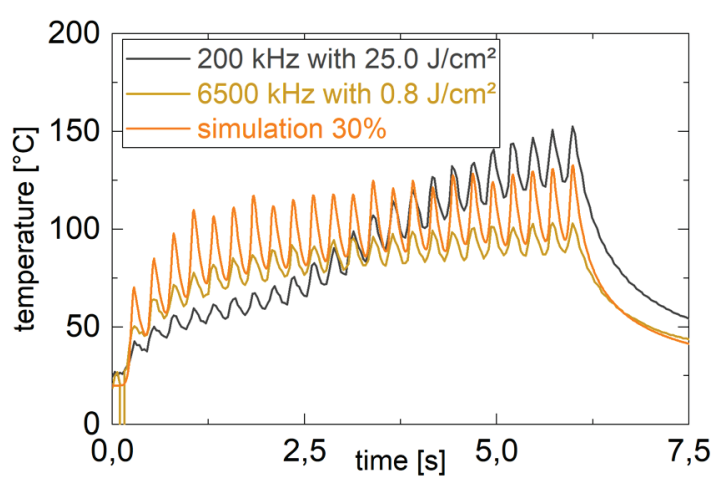

Fig. 7 Temperature development $0.82 \mathrm{~mm}$ below the surface for a processes with $70 \mathrm{~W}$ average power for stainless steel with $200 \mathrm{kHz}$ and $400 \mathrm{kHz}$ repetition rate and a simulation with $30 \%$ of the average laser power as a continuous surface heat source.

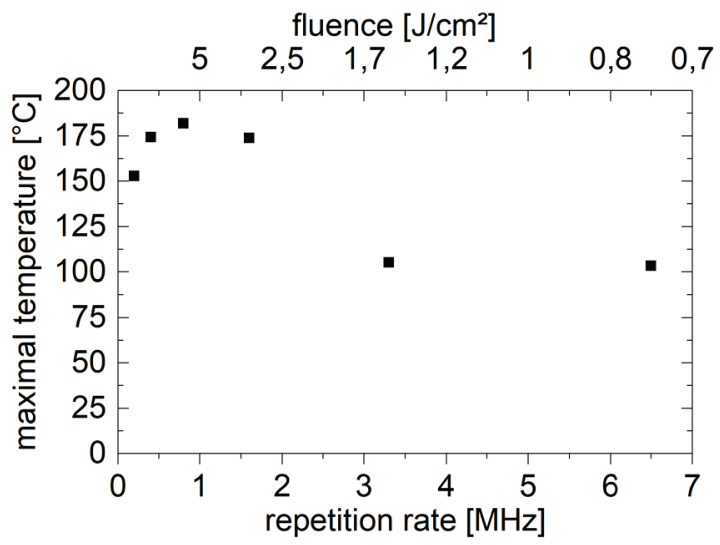

Fig. 8 Maximal temperature achieved within the $70 \mathrm{~W}$ processes.
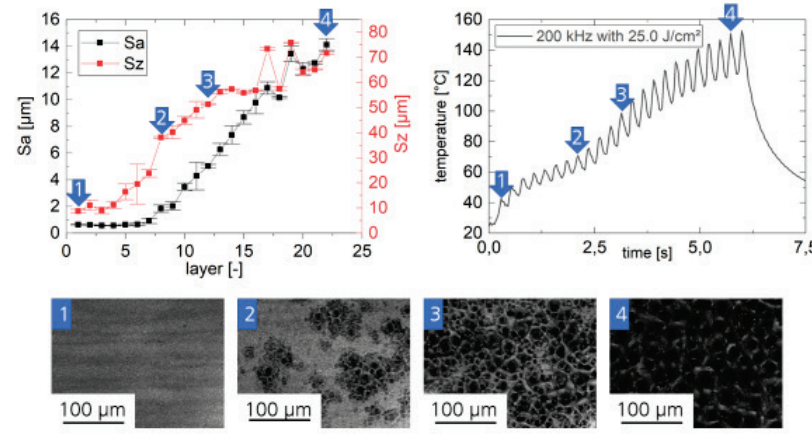

Fig. 9 Correlation of the ex-situ measured surface roughness and microscopic images to the thermal behavior during the $200 \mathrm{kHz}$ process with $70 \mathrm{~W}$ average power.
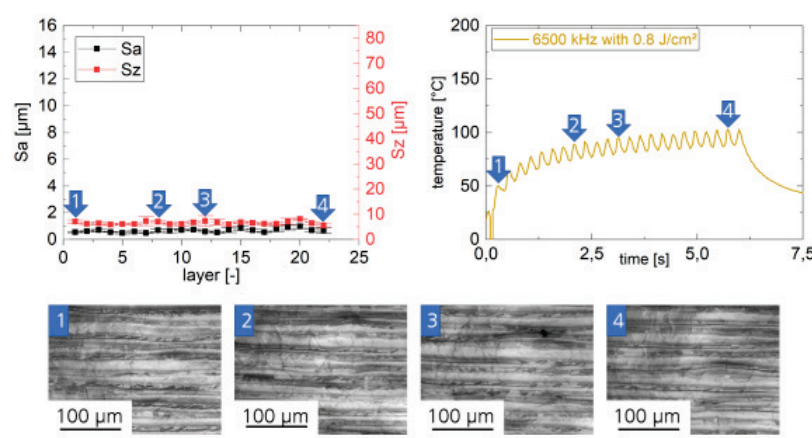

Fig. 10 Correlation of the ex-situ measured surface roughness and microscopic images to the thermal behavior during the $6500 \mathrm{kHz}$ process with $70 \mathrm{~W}$ average power. 

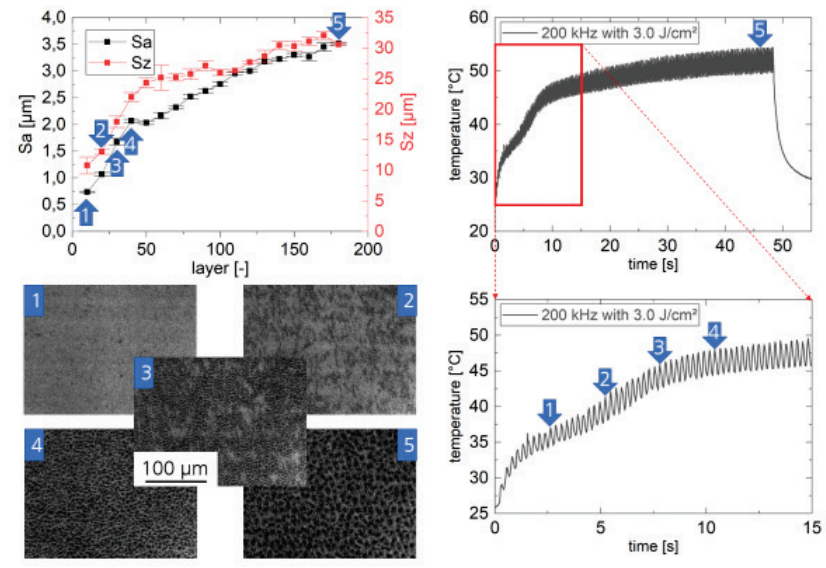

Fig. 11 Correlation of the ex-situ measured surface roughness and microscopic images to the thermal behavior during the $200 \mathrm{kHz}$ process with $8.5 \mathrm{~W}$ average power.
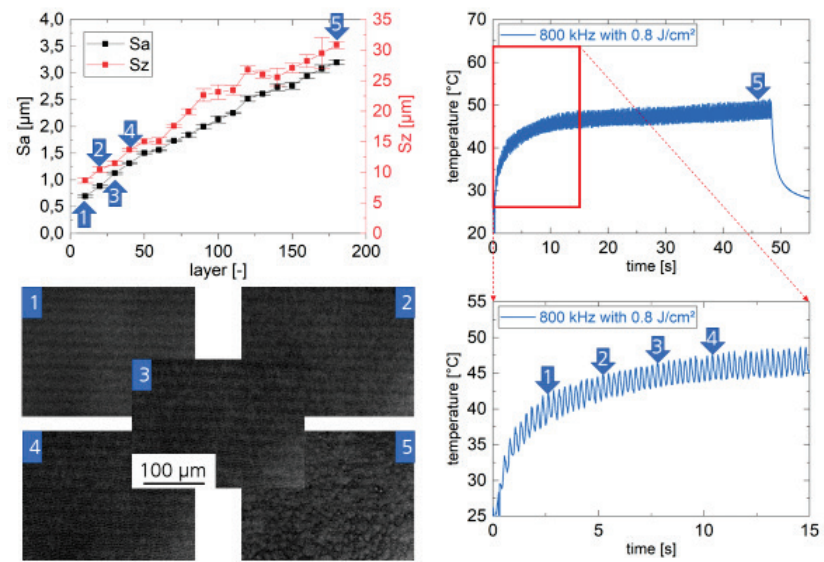

Fig. 12 Correlation of the ex-situ measured surface roughness and microscopic images to the thermal behavior during the $800 \mathrm{kHz}$ process with $8.5 \mathrm{~W}$ average power

an increased energy input due to multiple reflections on rough surface structures is assumed. In comparison, a high power process without the mentioned change in the thermal behavior is depicted in Fig. 10. There is no measurable increase in the surface roughness during the process. The surface morphology is dominated by a smooth molten layer from the beginning of the process to its end without significant change. This strengthens the presumption of the increased absorption due to the surface roughness.

Fig. 11 shows a similar development of the surface morphology and the surface roughness at the beginning of the process with $8.5 \mathrm{~W}$ average power and $200 \mathrm{kHz}$ repetition rate. Only the melt structures are less pronounced and conelike protrusions (CLPs) $[2,13]$ develop at the process end. Therefore, the assumption of the roughness induced absorption increase is the same for the low power processes and the nonevent rise of the temperature can be correlated to a change of the surface morphology.

Also for the low power processes an example without the change in thermal behavior during the process is evaluated (Fig. 12). The microstructure in the microscopic images is less pronounced compared to the measurements in Fig. 11 and no CLPs arise. Nevertheless, the roughness within the process increases steadily with a linear dependency to the number of passes. Due to no observable change in the

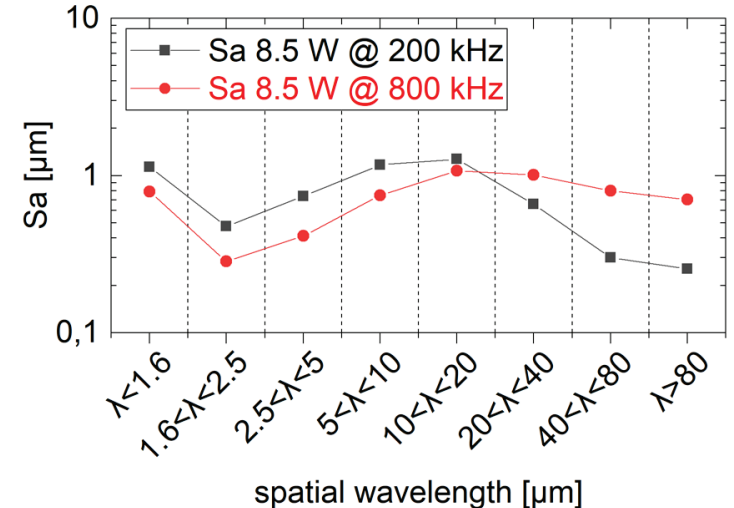

Fig. 13 Surface roughness of the $200 \mathrm{kHz}$ and $800 \mathrm{kHz}$ process for $8.5 \mathrm{~W}$ after 180 passes resolved by spatial wavelength.
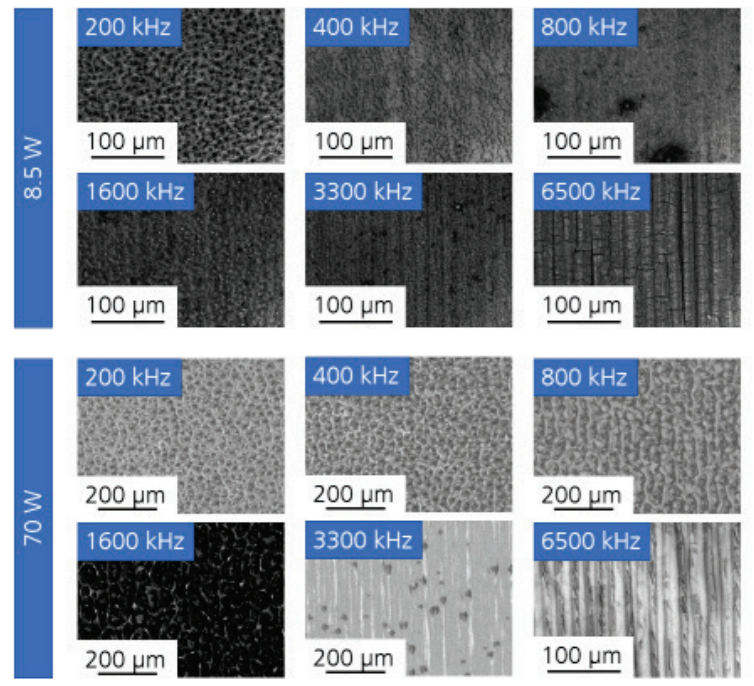

Fig. 14 Microscope images of the sample surfaces according to the observed processes.

thermal input but increasing surface roughness a closer examination of the surface roughness becomes necessary (Fig. 13).

In Fig. 13 the surface roughness of the $200 \mathrm{kHz}$ and the $800 \mathrm{kHz}$ process with $8.5 \mathrm{~W}$ after 180 passes of the scanning strategy is plotted versus the spatial wavelength. By comparing the roughness of the two processes it becomes evident that the processes with the linear roughness increase with a fluence next to the optimum ( $800 \mathrm{kHz}$ process) has a more pronounced roughness for high spatial wavelength, which is usually referred to as waviness. This is attributed to transferred macro structures of the scanning strategy and the spot size. Comparing the lower spatial wavelength leads to a measurable less pronounced micro surface roughness for the $800 \mathrm{kHz}$ process. The micro structures, which are responsible for the multiple reflections and the absorption increase and therefore for the change in the thermal behavior, are less pronounced here. This yields the constant thermal input.

Another indication, that the change of the thermal behavior during the processes is a surface effect, is gained from the comparison of the microscopic images of the surfaces morphology for all processes (Fig. 14). All processes that undergo a thermal change during the process time lead to a molten surface structure in the end. An exception to this, however, is the process with $6500 \mathrm{kHz}$ repetition rate and 
$8.5 \mathrm{~W}$ average power. For this process the used fluence is close to the reported ablation threshold for the used material [8]. So almost no ablation occurs and the whole absorbed energy heats up the surface, which is then oxidizing (Fig. 14).

\section{Conclusion}

To gain a better insight into heat accumulation during processing with ultrashort laser pulses and to test the theories discussed in literature in-situ thermography was performed. Contrary to the common opinion that high repetition rates lead to higher temperatures we show that residual heat and not the repetition rate is the key factor that determines the thermal load. Depending on the processing parameters high repetition rates can also lead to decreasing thermal load at a given average power. Furthermore, in contrast to the presented model for residual heat [7] based on the well-known threshold model $[1,5,6]$ and the most theoretical considerations and expectations $[3,7,9,11,14]$, we show that the residual heat cannot be assumed to be a constant parameter.

This conclusion must be drawn because some processes are observed to change in thermal behavior during the process. In the thermographic measurements this finds expression in an uneven rise of the workpiece temperature. These processes are identified with parameters outside the usual machining range, which means the used fluence is not close to the optimum fluence derived from the theory $[5,6,8]$ which is determined experimentally for these evaluation (cf. Fig. 4).

For the regime next to the optimum fluence a constant heat input is a good approximation. A comparison with a simulation of heat accumulation was carried out. For these simulation a constant heat source with $30 \%$ of the applied power $(70 \mathrm{~W})$ is used to calculate the temperature distribution over time. For the two processes next to the optimum fluence a good agreement between experimental data and the simulation in temperature level and profile is observed. Consequently, a factor $\eta_{\text {res }} \approx 0.3$ seems to be a good estimation for the residual heat input for these processes which is $10 \%$ below the value determined by Bauer et al. [11]. But this reduction may be an effect of shielding effects due to the high repetition rates as described by Finger et al. [7]. For the other processes with parameters that deviate strongly from the optimum, the thermal change is not mapped by the theory. If the process is not evaluated in-situ, e.g. by means of the final temperature of the workpiece, this would lead to an incorrect estimation of a constant residual heat share.

For further investigations of the thermal change that occurs during some processes the in-situ analysis is supplemented by an ex-situ analysis of the surface condition in between the layers of the scanning strategy. During the processes CLPs $[2,13](8.5 \mathrm{~W})$ or rather melting structures $(70 \mathrm{~W})$ are formed on the sample surface due to heat accumulation at the surface. The occurrence of these micro structures is correlated with the increase of the temperature in the workpiece over time. Furthermore, for processes with no change in the thermal input these micro structures are less pronounced or absent. Therefore, an absorption increase by multiple reflection within micro structures is identified as reason for the higher heat accumulation. Therefore, this is a kind of self-reinforcing effect within certain limits.

Another indication for this effect is the surface morphology after the process end shown in Fig. 14. All processes with a change in the temperature curve during the process show a rough and molten surface finish in the end. The only exception to this is the process with a fluence close to the ablation threshold. In this case almost no ablation occurs and the heat accumulation lead to an oxidation of the surface $(6500 \mathrm{kHz}$ with $8.5 \mathrm{~W})$. For this parameters close to the ablation threshold further investigations must be carried out.

As a consequence of these results, it is possible to design processes and estimate the heat input with a constant heat source in a operating area in the proximity of the optimum fluence.

For a better validation of the residual heat model based on the threshold model, further investigations and evaluations, which are not presented in this work, regarding the ablation efficiency have to be carried out in order to investigate further restrictions regarding the permissible parameter space due to shielding effects that will have a influence that is not negligible for high repetition rates.

\section{Acknowledgments and Appendixes}

The authors would like to thank the German Research Foundation DFG for funding this work under Grant No. PO 591/41-1.

\section{References}

[1] S. Nolte, C. Momma, H. Jacobs, A. Tünnermann, B. N. Chichkov, B. Wellegehausen, and H. Welling: J. Opt. Soc. Am. B, 14, (1997) 2716.

[2] J. Schille, L. Schneider, A. Streek, S. Kloetzer, and U. Loeschner: Opt. Eng, 55, (2016) 96109.

[3] J. Finger and M. Reininghaus: Opt. Express, 22, (2014) 18790.

[4] T. Kramer, B. Neuenschwander, B. Jäggi, S. Remund, U. Hunziker, and J. Zürcher: Physics Procedia, 83, (2016) 123.

[5] G. Raciukaitis: J. Laser Micro/Nanoengin, 4, (2009) 186.

[6] B. Neuenschwander, G. F. Bucher, C. Nussbaum, B. Joss, M. Muralt, U. W. Hunziker, and P. Schuetz, Proc. SPIE, Vol. 7584, (2010) 75840R.

[7] J. Finger, B. Bornschlegel, M. Reininghaus, A. Dohrn, M. Nießen, A. Gillner, and R. Poprawe: Adv. Opt. Technol., 7, (2018) 145.

[8] B. Jaeggi, B. Neuenschwander, M. Zimmermann, L. Penning, R. deLoor, K. Weingarten, and A. Oehler, Proc. SPIE, Vol. 8967, (2014) 89670Q.

[9] B. Neuenschwander, T. Kramer, B. Lauer, and B. Jaeggi, Proc. SPIE, Vol. 9350, (2015) 93500U.

[10] B. Jäggi, L. Cangueiro, D. Brunell, J. A. Ramos de Campos, C. Hairaye, and B. Neuenschwander, Proc. SPIE, Vol. 10519, (2018) 1051905.

[11] F. Bauer, A. Michalowski, T. Kiedrowski, and S. Nolte: Opt. Express, 23, (2015) 1035.

[12] B. Jäggi, D. J. Förster, R. Weber, and B. Neuenschwander: Adv. Opt. Technol., 7, (2018) 175.

[13] S. Brüning, G. Jenke, K. Du, and A. Gillner: Physics Procedia, 56, (2014) 919.

[14] R. Weber, T. Graf, P. Berger, V. Onuseit, M. Wiedenmann, C. Freitag, and A. Feuer: Opt. Express, 22, (2014) 11312.

(Received: May19, 2019, Accepted: May26, 2020) 